\title{
Association between single nucleotide polymorphisms in ADRB2, GNB3 and GSTP1 genes and high-altitude pulmonary edema (HAPE) in the Chinese Han population
}

\author{
Yongjun $\mathrm{He}^{1,2,3, *}$, Lijun Liu' ${ }^{1,2,3, *}$, Pengcheng $\mathrm{Xu}^{4}$, $\mathrm{Na} \mathrm{He}{ }^{1,2,3}$, Dongya Yuan ${ }^{1,2,3}$, Longli \\ Kang $^{1,2,3}$, Tianbo Jin $^{1,2,3}$ \\ ${ }^{1}$ Key Laboratory of High Altitude Environment and Genes Related to Diseases of Tibet Autonomous Region, School of \\ Medicine, Xizang Minzu University, Xianyang, Shaanxi 712082, China \\ ${ }^{2}$ Key Laboratory for Molecular Genetic Mechanisms and Intervention Research on High Altitude Disease of Tibet Autonomous \\ Region, School of Medicine, Xizang Minzu University, Xianyang, Shaanxi 712082, China \\ ${ }^{3}$ Key Laboratory for Basic Life Science Research of Tibet Autonomous Region, School of Medicine, Xizang Minzu University, \\ Xianyang, Shaanxi 712082, China \\ ${ }^{4}$ Department of Hand Surgery, Hebei Province Cangzhou Hospital of Integrated Traditional and Western Medicine, Cangzhou, \\ Hebei 061001, China \\ *These authors have contributed equally to this work \\ Correspondence to: Tianbo Jin, email: jintianbo@gmail.com
}

Keywords: GNB3, GSTP1, ADRB2, high-altitude pulmonary edema, genetic

Received: August 12, 2016

Accepted: December 07, 2016

Published: February 14, 2017

\section{ABSTRACT}

High altitude pulmonary edema (HAPE) occurs mainly under conditions such as high altitude, rapid ascent, or hypoxia. Previous studies suggest that ADRB2, GNB3, TH, and GSTP1 polymorphisms are associated with various lung diseases. We evaluated whether those polymorphisms are associated with the risk of HAPE in a Chinese Han population. ADRB2, GNB3, TH and GSTP1 polymorphisms were genotyped using a Sequenom MassARRAY. Logistic regression, adjusted for age and gender, was used to evaluate the association between the genotypes and the risk of HAPE by computing odds ratios (ORs) and $95 \%$ confidence intervals ( $95 \% \mathrm{CIs}$ ). The results revealed that GNB3 rs4963516 allele "G" (G vs T: OR $=0.70,95 \%$ CI $=0.55-$ $0.90, p=0.006$ ) was associated with HAPE risk. The ADRB2 rs1042718 alleles had a 1.29 -fold $(95 \% \mathrm{CI}=1.00-1.66 ; p=0.045)$ increased risk of HAPE, and the GSTP1 rs749174 alleles had a 0.71 -fold $(95 \% \mathrm{CI}=0.52-0.99 ; p=0.042)$ decreased risk of HAPE. Co-dominant and dominant models of GNB3 rs4963516 decreased the risk of HAPE ( $p=0.023$ and $p=0.008$, respectively). Our results indicate GNB3 and GSTP1 polymorphisms may protect against HAPE progression, while ADRB2 polymorphisms are associated with an increased risk of HAPE.

\section{INTRODUCTION}

High-altitude pulmonary edema (HAPE), a potentially fatal medical condition, is the cause of deaths due to high-altitude illness [1]. HAPE is a form of increased permeability pulmonary edema of noncardiogenic origin that usually occurs within 2-4 days of ascent above 2,500-3,000 m [1,2]. Patients with HAPE include two types: inhabitants of high-altitudes who return to low altitudes, also known as "re-entry" HAPE, and healthy lowlanders who rapidly climb up and are not acclimatized to high altitudes [3]. In HAPE, hypoxia-induced pulmonary vasoconstriction leads to endothelial dysfunction and intravascular fluid retention $[4,5]$. HAPE occurs frequently in people exposed to high altitudes $[6,7]$. More than 140 million people living in high-mountain areas, including Asia and Americas, have suffered from HAPE [3]. Recently, the HAPE mortality rates have reached $50 \%$ [8], because of the increase in skiing, trekking, and climbing tours.

HAPE is a multifactorial disease that involves both environmental and genetic factors. Studies indicate that cell and tissue hypoxia can promote gene expression and induce expression of physiologically important proteins 
Table 1: Characteristics of the subjects

\begin{tabular}{|c|c|c|c|}
\hline variable & case & control & $p$ \\
\hline Number & 267 & 304 & \\
\hline sex & & & $>0.001$ \\
\hline male & 246 & 290 & \\
\hline female & 21 & 14 & \\
\hline age, year (mean $\pm \mathrm{SD})$ & $32.57 \pm 10.74$ & $36.15 \pm 4.45$ & $<0.001^{*}$ \\
\hline
\end{tabular}

${ }^{*} p<0.05$ indicates statistical significance

Table 2: Allele frequencies in cases and controls and odds ratio estimates for HAPE risk

\begin{tabular}{|c|c|c|c|c|c|c|c|c|c|c|}
\hline SNP & Gene & Band & $\begin{array}{c}\text { Base } \\
\text { Change }\end{array}$ & MAF-case & $\begin{array}{l}\text { MAF- } \\
\text { control }\end{array}$ & $\begin{array}{l}p \text { value for } \\
\text { HWE test }\end{array}$ & OR & \multicolumn{2}{|c|}{$95 \%$ CI } & $p$ value \\
\hline rs17778257 & $A D R B 2$ & $5 q 32$ & $\mathrm{~A} / \mathrm{T}$ & 0.36 & 0.35 & 0.38 & 1.03 & 0.81 & 1.31 & 0.827 \\
\hline rs2400707 & $A D R B 2$ & $5 q 32$ & $\mathrm{~A} / \mathrm{G}$ & 0.29 & 0.34 & 0.80 & 0.81 & 0.63 & 1.04 & 0.093 \\
\hline rs17108803 & $A D R B 2$ & $5 q 32$ & $\mathrm{G} / \mathrm{T}$ & 0.05 & 0.05 & 0.48 & 0.98 & 0.56 & 1.71 & 0.940 \\
\hline rs 12654778 & $A D R B 2$ & $5 q 32$ & $\mathrm{~A} / \mathrm{G}$ & 0.36 & 0.34 & 0.31 & 1.06 & 0.83 & 1.35 & 0.642 \\
\hline rs 11168070 & $A D R B 2$ & $5 q 32$ & $\mathrm{C} / \mathrm{G}$ & 0.09 & 0.09 & 0.73 & 0.95 & 0.63 & 1.43 & 0.815 \\
\hline rs 1042718 & $A D R B 2$ & $5 q 32$ & $\mathrm{~A} / \mathrm{C}$ & 0.36 & 0.30 & 0.68 & 1.29 & 1.01 & 1.67 & $0.045^{\star}$ \\
\hline rs3842727 & $T H$ & $11 \mathrm{p} 15.5$ & $\mathrm{G} / \mathrm{T}$ & 0.04 & 0.04 & 1.00 & 0.95 & 0.52 & 1.73 & 0.860 \\
\hline rs2070762 & $T H$ & $11 \mathrm{p} 15.5$ & $\mathrm{~A} / \mathrm{G}$ & 0.39 & 0.36 & 0.14 & 1.13 & 0.89 & 1.44 & 0.311 \\
\hline rs 10770140 & $T H$ & $11 \mathrm{p} 15.5$ & $\mathrm{C} / \mathrm{T}$ & 0.09 & 0.09 & 1.00 & 1.10 & 0.74 & 1.66 & 0.632 \\
\hline rs 10770141 & $T H$ & $11 \mathrm{p} 15.5$ & $\mathrm{~A} / \mathrm{G}$ & 0.08 & 0.08 & 0.24 & 1.00 & 0.66 & 1.53 & 0.992 \\
\hline rs10840491 & $T H$ & $11 \mathrm{p} 15.5$ & $\mathrm{~A} / \mathrm{G}$ & 0.10 & 0.09 & 1.00 & 1.11 & 0.74 & 1.66 & 0.611 \\
\hline rs7119275 & $T H$ & $11 \mathrm{p} 15.5$ & $\mathrm{C} / \mathrm{T}$ & 0.09 & 0.09 & 0.15 & 1.06 & 0.70 & 1.59 & 0.795 \\
\hline rs749174 & GSTP1 & $11 \mathrm{q} 13.2$ & $\mathrm{~A} / \mathrm{G}$ & 0.14 & 0.18 & 0.85 & 0.72 & 0.52 & 0.99 & $0.042^{*}$ \\
\hline rs4963516 & GNB3 & $12 \mathrm{p} 13.31$ & $\mathrm{G} / \mathrm{T}$ & 0.29 & 0.37 & 0.27 & 0.70 & 0.55 & 0.90 & $0.006^{*}$ \\
\hline rs5446 & GNB3 & $12 \mathrm{p} 13.31$ & $\mathrm{C} / \mathrm{T}$ & 0.20 & 0.20 & 0.15 & 1.02 & 0.76 & 1.36 & 0.914 \\
\hline
\end{tabular}

$\mathrm{MAF}=$ minor allele frequency; HWE $=$ Hardy-Weinberg Equilibrium; OR $=$ odds ratio; $95 \% \mathrm{CI}=95 \%$ confidence interval; ${ }^{*} p<0.05$ indicates statistical significance

[9-13]. The genetic sensitivity to hypoxia has been known for years, and includes genes, such as nitric oxide synthase (NOS) [9], tyrosine hydroxylase (TH) [14], angiotensinconverting enzyme (ACE) [10], endothelin-1 (EDN-1) [11, 12], and vascular endothelial growth factor (VEGF) [13]. Genetic single nucleotide polymorphisms (SNPs) play a pivotal role in high altitude diseases, including the HAPE risk [15]. However, genetic studies about the etiology of HAPE are still rare.

In our study, we selected $15 \mathrm{SNPs}$ in beta-2 adrenergic receptor (ADRB2), $G$ protein $\beta 3$ subunit (GNB3), glutathione transferase Pi 1 (GSTP1), and TH genes, which have been reported to be associated with pulmonary diseases, including asthma, HAPE, lung cancer, and chronic obstructive pulmonary disease (COPD) [1620]. Based on previous research, we conducted a casecontrol study and identified an association of the $A D R B 2$, $G N B 3, T H$, and GSTP1 genes with the risk of HAPE in Chinese Han population. These results may provide a basis for the clinical prevention of HAPE.

\section{RESULTS}

A total of 267 patients with HAPE and 304 healthy people were enrolled in this case-control study. The HAPE cases and controls were matched by sex, but there was a significant difference in age between HAPE cases and controls $(p<0.05)$; the average ages were $36.2 \pm 4.5$ years 
Table 3: Single-SNP analysis (rs1042718, rs749174 and rs4963516) under different genetic models (adjusted by age and gender)

\begin{tabular}{|c|c|c|c|c|c|c|}
\hline SNP & Model & Genotype & control & case & OR $(95 \%$ CI $)$ & $p$ \\
\hline \multirow[t]{8}{*}{ rs 1042718} & \multirow{3}{*}{ Co-dominant } & $\mathrm{C} / \mathrm{C}$ & $144(49 \%)$ & $110(43.5 \%)$ & 1 & \multirow{3}{*}{0.140} \\
\hline & & $\mathrm{C} / \mathrm{A}$ & $121(41.2 \%)$ & $103(40.7 \%)$ & $1.12(0.77-1.62)$ & \\
\hline & & $\mathrm{A} / \mathrm{A}$ & $29(9.9 \%)$ & $40(15.8 \%)$ & $1.76(1.01-3.06)$ & \\
\hline & \multirow{2}{*}{ Dominant } & $\mathrm{C} / \mathrm{C}$ & $144(49 \%)$ & $110(43.5 \%)$ & 1 & \multirow{2}{*}{0.230} \\
\hline & & $\mathrm{C} / \mathrm{A}-\mathrm{A} / \mathrm{A}$ & $150(51 \%)$ & $143(56.5 \%)$ & $1.24(0.88-1.76)$ & \\
\hline & \multirow{2}{*}{ Recessive } & $\mathrm{C} / \mathrm{C}-\mathrm{C} / \mathrm{A}$ & $265(90.1 \%)$ & $213(84.2 \%)$ & 1 & \multirow{2}{*}{0.056} \\
\hline & & $\mathrm{A} / \mathrm{A}$ & $29(9.9 \%)$ & $40(15.8 \%)$ & $1.67(0.98-2.82)$ & \\
\hline & Log-additive & --- & --- & --- & $1.26(0.98-1.62)$ & 0.072 \\
\hline \multirow[t]{8}{*}{ rs4963516 } & \multirow{3}{*}{ Co-dominant } & $\mathrm{T} / \mathrm{T}$ & $126(41.7 \%)$ & $138(51.7 \%)$ & 1 & \multirow{3}{*}{0.023} \\
\hline & & $\mathrm{G} / \mathrm{T}$ & $131(43.4 \%)$ & $104(39 \%)$ & $0.66(0.46-0.95)$ & \\
\hline & & $\mathrm{G} / \mathrm{G}$ & $45(14.9 \%)$ & $25(9.4 \%)$ & $0.54(0.31-0.93)$ & \\
\hline & \multirow{2}{*}{ Dominant } & $\mathrm{T} / \mathrm{T}$ & $126(41.7 \%)$ & $138(51.7 \%)$ & 1 & \multirow{2}{*}{0.008} \\
\hline & & G/T-G/G & $176(58.3 \%)$ & $129(48.3 \%)$ & $0.63(0.45-0.89)$ & \\
\hline & \multirow{2}{*}{ Recessive } & $\mathrm{T} / \mathrm{T}-\mathrm{G} / \mathrm{T}$ & $257(85.1 \%)$ & $242(90.6 \%)$ & 1 & \multirow{2}{*}{0.110} \\
\hline & & $\mathrm{G} / \mathrm{G}$ & $45(14.9 \%)$ & $25(9.4 \%)$ & $0.65(0.38-1.10)$ & \\
\hline & Log-additive & --- & --- & --- & $0.71(0.55-0.91)$ & 0.110 \\
\hline \multirow[t]{8}{*}{ rs749174 } & \multirow{3}{*}{ Co-dominant } & $\mathrm{G} / \mathrm{G}$ & $201(66.8 \%)$ & $198(74.2 \%)$ & 1 & \multirow{3}{*}{0.190} \\
\hline & & $\mathrm{A} / \mathrm{G}$ & $91(30.2 \%)$ & $65(24.3 \%)$ & $0.74(0.51-1.09)$ & \\
\hline & & $\mathrm{A} / \mathrm{A}$ & $9(3 \%)$ & $4(1.5 \%)$ & $0.50(0.15-1.66)$ & \\
\hline & \multirow{2}{*}{ Dominant } & $\mathrm{G} / \mathrm{G}$ & $201(66.8 \%)$ & $198(74.2 \%)$ & 1 & \multirow{2}{*}{0.086} \\
\hline & & $\mathrm{A} / \mathrm{G}-\mathrm{A} / \mathrm{A}$ & $100(33.2 \%)$ & $69(25.8 \%)$ & $0.72(0.50-1.05)$ & \\
\hline & \multirow{2}{*}{ Recessive } & $\mathrm{G} / \mathrm{G}-\mathrm{A} / \mathrm{G}$ & $292(97 \%)$ & $263(98.5 \%)$ & 1 & \multirow{2}{*}{0.300} \\
\hline & & $\mathrm{A} / \mathrm{A}$ & $9(3 \%)$ & $4(1.5 \%)$ & $0.54(0.16-1.79)$ & \\
\hline & Log-additive & --- & --- & --- & $0.73(0.52-1.03)$ & 0.067 \\
\hline
\end{tabular}

OR $=$ odds ratio $; 95 \% \mathrm{CI}=95 \%$ confidence interval;

${ }^{*} p<0.05$ indicates statistical significance

and $32.6 \pm 10.7$ years, respectively. The characteristics of the studied population are summarized in Table 1.

Table 2 summarizes the basic information of candidate SNPs in our study, such as chromosomal position, gene, allele, Hardy-Weinberg equilibrium (HWE) test results, and minor allele frequency (MAF). The HWE was agreed by $p>0.05$. All success rates of the genotype assays were designed to have the cut off level less $98 \%$.

We compared the frequency of alleles distributions and found that three SNPs were associated with HAPE risk in three genes (rs1042718 in ADRB2; OR $=1.29,95$ $\% \mathrm{CI}=1.00-1.67, p=0.044 ;$ rs749174 in GSTP1; OR $=$ $0.71,95 \% \mathrm{CI}=0.52-0.99, p=0.041$; and $\mathrm{rs} 4963516$ in $G N B 3 ; \mathrm{OR}=0.70,95 \% \mathrm{CI}=0.55-0.90, p=0.006)$.
The results of the genetic model are shown in Table 3. Co-dominant, dominant, recessive, and logadditive models were calculated and adjusted by age and gender. We found that co-dominant ( $p=0.023)$ and dominant models (GT/GG vs TT: OR $=0.63$, $95 \% \mathrm{CI}=0.45-0.89, p=0.008)$ in $G N B 3 \mathrm{rs} 4963516$ were associated with the risk of HAPE. We did not find any statistically significant association between the genotype and genetic model in other SNPs and the risk of HAPE. No association was observed after Bonferroni correction.

Finally, in order to assess the association between SNP haplotypes and HAPE risk, a Wald test was performed using an unconditional multivariate regression analysis. However, no association was observed (data not shown). 
Table 4: The SNPs primers used for this study

\begin{tabular}{|c|c|c|c|}
\hline SNP & 1st_PCRP & 2st_PCRP & UEP_SEQ \\
\hline rs 17778257 & ACGTTGGATGCAGTTTTTCAAAGACACCAC & ACGTTGGATGCTTTCCATCTGGCATGTGAC & GTGAAATTAATTTTCAGGGTTTGA \\
\hline rs2400707 & ACGTTGGATGTAAGTCACAGACGCCAGATG & ACGTTGGATGTCCTTTCATCTGCTGGATAG & GTTTGTTAATCTTTCGGGTTG \\
\hline rs 17108803 & ACGTTGGATGAAGCACAAAGACATGGTGAC & ACGTTGGATGTGTCTGTGTCGCTCTGGATG & ccctCCAGCGTGTGTTTACTT \\
\hline rs 12654778 & ACGTTGGATGGTGTGTCTCAGTGTCTATGG & ACGTTGGATGAGGCACAAATACACCCTGGC & ccetcCACCCTGGCAGACATGCT \\
\hline rs 11168070 & ACGTTGGATGACAGAAGAGCCAAAAGCTCC & ACGTTGGATGAGAGGGCTAAAGCTGGAGG & gaTAAAGCTGGAGGTGGTGT \\
\hline rs1042718 & ACGTTGGATGTGAAGAAGTCACAGCAGGTC & ACGTTGGATGTCTTGCCCATTCAGATGCAC & attgaCCATTCAGATGCACTGGTAC \\
\hline rs3842727 & ACGTTGGATGAGCCACGTGACAGTGGGAG & ACGTTGGATGAGCCAGCACAGTTTGTGACC & TGTGACCCCTGCTCCCT \\
\hline rs2070762 & ACGTTGGATGTCCTTCTCACGGATGGTGTC & ACGTTGGATGTCACAGATGAGAAAACCGAC & TGAGAAAACCGACCCCTGG \\
\hline rs 10770140 & ACGTTGGATGTAGGAGTGCCATCTGCCCA & ACGTTGGATGCCCTCCTGGGACATTCTGG & ttttGGACATTCTGGACCCCAC \\
\hline rs10770141 & ACGTTGGATGTCTCCAAGGGGAAGGCATCA & ACGTTGGATGACTGCTAGCTCCTGGCTTC & CCTGTGGCCCCTTCTTT \\
\hline rs10840491 & ACGTTGGATGAGAGTGGGCCCTGAGAGATG & ACGTTGGATGAAGCCCTAGACGCTCCCTGA & AGACGCTCCCTGACTTCTC \\
\hline rs7119275 & ACGTTGGATGCACTGGGTGCTGAGAGACA & ACGTTGGATGTGCCACGATATTAATGCCCC & сCCCCTCCCTTGCGCTCC \\
\hline rs749174 & ACGTTGGATGTTCAGACTTCTCAATGGCCC & ACGTTGGATGAGGTCCCGAAGGCCTTGAA & tcttTCCCGAAGGCCTTGAACCCACT \\
\hline rs4963516 & ACGTTGGATGTCTCGCTACCACTTCCTACT & ACGTTGGATGTGCTCTGCAAGTTGAAGCTG & ccccaATTTGCCCCAAGCTAGTCCC \\
\hline rs5446 & ACGTTGGATGTGGGTGGTATAGGGCGTTTG & ACGTTGGATGAGCATGAATAAGAAGAGGGC & ccccAGAAGAGGGCCAGGACCCTAGT \\
\hline
\end{tabular}

\section{DISCUSSION}

In this study, we hypothesized that the $A D R B 2$, GNB3, TH, and GSTP1 polymorphisms are associated with the HAPE risk in Chinese Han population. Our results show that the alleles "G" in GNB3 rs4963516 $(p=0.006)$, "A" in ADRB2 rs1042718 $(p=0.045)$, and "A" in GSTP1 rs749174 $(p=0.041)$ are associated with the risk of HAPE.

The GNB3 gene, located in 12p13, has been associated with mountain sickness [17]. It has been also reported in the hypertensive crisis, cardiovascular disease, and digestive system cancer [21, 22]. GNB3 regulates heterotrimeric guanine nucleotide-binding proteins (G-proteins), which integrate signals between receptors and effector proteins. HAPE is characterized by increased water and salt metabolism, and by abnormal cell membrane ion transport, which are regulated by G-proteins and beta 2 adrenergic receptor protein. In this study, we found that co-dominant and dominant models of GNB3 rs4963516 are associated with the decreased risk of HAPE. In contrast, we did not find a statistically significant correlation between rs5446 in GNB3 gene and the risk of HAPE; this is consistent with a previous study [23].

We used Hap Map to identify SNPs in the ADRB2 gene, a small (3446bp transcript, 1239bp coding sequence), intronless gene on chromosome 5 (Gene-Card number GC05P148186), which is the principal catecholamine receptor in the lungs, and plays a crucial role in pulmonary diseases. Wang et al reported that the common haplotypes in the $A D R B 2$ are not associated with acute mountain sickness susceptibility in Nepalese population [24], while
Chandramoorthi et al showed a significant association between the $A D R B 2$ gene and the HAPE risk [25]. In this study, we found that the allelic model in ADRB2 rs 1042718 is associated with the risk of HAPE (Table 2). However, our results show that the genetic models of $A D R B 2$ are not associated with the risk of HAPE.

GSTP1 gene has been associated with various cancers, including breast, lung, prostate, bladder, and esophageal cancer [26]. In addition, Mishra et al have shown a correlation between the GSTP1 gene and the risk of HAPE [27]. Our study indicates that the polymorphism of GSTP1 rs 749174 is associated with the risk of HAPE.

Consistent with previous studies, we have found no significant correlation between the blunted hypervariable region values in HAPE with the polymorphisms of the $T H$ gene. These results indicate that the pathogenesis of HAPE is not influenced by the mutations of the $T H$, and that these polymorphisms may not be suitable genetic markers for the HAPE-susceptibility.

A limitation of this study is the relatively small data set, which limits the statistical power to detect effects for several of the assessed SNPs, particularly since much of them are not frequent. In addition, while the potential risk factors for HAPE, such as body mass index, menopausal status, smoking, and age, may interact with the SNPs, the relative similarity of our patient and control populations has reduced any confounding effects. Using Bonferroni correction, no statistically significant association between SNPs and the risk of HAPE has been found. This may be due to the relatively small sample size, the selection criteria for SNPs (minor allele frequency $>5 \%$ ), and the weakness of the Bonferroni correction itself (the result 
interpretation depends on the number of other tests performed). True differences may have been deemed nonsignificant given the likelihood of type II errors.

To further evaluate the effect of polymorphisms of $A D R B 2, G N B 3$ and GSTP1 genes on the HAPE risk, future studies should analyze gene-gene and gene-environment interactions using large sample sizes.

\section{CONCLUSION}

This study demonstrates the association between the risk of HAPE and the polymorphism of $A D R B 2, G N B 3, T H$, and GSTP1 genes. Polymorphisms of GNB3 and GSTP1 genes may be a potential protective factor in the HAPE progression in Chinese Han population. In contrast, $A D R B 2$ may increase the risk of HAPE. Additional studies are needed to confirm this association, and clarify the role of $A D R B 2$, GNB3 and GSTP1 genes in the HAPE pathogenesis.

\section{MATERIALS AND METHODS}

\section{Study participants}

Of the 571 participants, 267 were diagnosed with HAPE and recruited between August 2013 and December 2015 at the Hospital of the School of Medicine, Xizang Minzu University, China. The key inclusion criteria were based on clinical symptoms, epidemiology, and pathophysiology findings [28]. The clinical features recorded were: age at diagnosis, gender, radiological results, chest sounds, body temperature, heart rate, and oxygen saturation. All HAPE patients eventually exhibited chest infiltrates consistent with pulmonary edema. Controls $(n=304)$ were healthy people selected from the same geographic region as the HAPE cases and recruited from the Hospital of the School of Medicine, Xizang Minzu University, China. The controls did not develop any symptoms or signs of HAPE or any high pressurerelated diseases after exposure to high altitude within 7 days. All participants were of Chinese Han population and resided at low altitudes less than $2000 \mathrm{~m}$. No participants used prophylactic medications, and the rate and altitude of ascent were the same among the HAPE cases and controls (altitude of the Tibetan plateau is 4000 to $5000 \mathrm{~m}$ ). Venous blood samples were obtained from the same hospital to avoid definite selection bias, and the blood samples were taken according to the study protocol approved by the Ethics Committee of Mental Health Center, from the Hospital of School of Medicine, Xizang Minzu University. Informed consent for genetic testing was obtained from each participant before the enrollment of the subjects.

\section{SNP selection and genotyping}

We selected 15 putative functional SNPs in $A D R B 2$, GNB3, TH and GSTP1 genes. The SNPs were found from recent respiratory publications. The first report was an acute mountain sickness susceptibility study in Nepalese population; from that study, SNPs in $A D R B 2$ (rs2400707, rs12654778, rs11168070, and rs1042718) were selected [29]. The second report suggested that the $T H$ gene may be associated with homovanillic acid and 3-methoxy-4-hydroxyphenylglycol, and the SNPs selected were rs10770140, rs10770141, and rs10840491 [30]. Finally, other studies identified the remaining SNPs associated with lung function [27, 31-33]. The SNPs were identified using dbSNP database (http:// www.ncbi.nlm.nih.gov/projects/SNP), and genotyped using Sequenom MassARRAY RS1000 (Sequenom, Inc) following the manufacturer's instructions. Primers were designed for each SNP and are listed in Table 4. Genotyping and data management were performed at the Molecular Research Laboratory of the Biotechnology, Northwest University, China. The overall success rate of all the genotyping assays was over $98 \%$. All samples with ambiguous results were measured repeatedly, as was a random selection of $10 \%$ of all samples to ensure laboratory quality control.

\section{Statistical analysis}

Statistical analyses were performed using SPSS version 17.0 for Windows (SPSS, Chicago, IL) and SNPstats software platform (http://bioinfo.iconcologia. net/SNPstats_web). Analysis of variance (ANOVA) was used to compare differences between genotypes for the various parameters. Chi-square test was used to identify departures from Hardy-Weinberg equilibrium. Analysis for genotype and allele effects was undertaken using the Chi-square test of independence, OR and 95\% CI were calculated from the combined results of both populations, adjusting for age and gender. In addition, comparison of population characteristics was performed using an independent sample t-test (using Levene's test for equality of variances) for continuous variables and an additional Chi-square test for categorical variables.

\section{ACKNOWLEDGMENTS}

This work was supported by the Major science and technology research projects of Xizang (Tibet) Autonomous Region (2015XZ01G23), and National Natural Science Foundations (No. 81560516). The authors wish to thank participants in the study who made this work possible. We would also like to thank the clinicians and other hospital staff who contributed to the blood sample and data collection for this study.

\section{CONFLICTS OF INTEREST}

All authors declare no conflict of interest. 


\section{REFERENCES}

1. West JB. High-altitude medicine. American journal of respiratory and critical care medicine. 2012; 186:1229-1237.

2. Maggiorini M. High altitude-induced pulmonary oedema. Cardiovasc Res. 2006; 72:41-50.

3. Bhagi S, Srivastava S and Singh SB. High-altitude pulmonary edema: review. J Occup Health. 2014; 56:235-243.

4. Hackett PH and Roach RC. High-altitude illness. N Engl J Med. 2001; 345:107-114.

5. Garnier J, Osguthorpe DJ and Robson B. Analysis of the accuracy and implications of simple methods for predicting the secondary structure of globular proteins. J Mol Biol. 1978; 120:97-120.

6. Hackett $\mathrm{P}$ and Rennie D. High-altitude pulmonary edema. Jama. 2002; 287:2275-2278.

7. Vock P, Fretz C, Franciolli M and Bartsch P. Highaltitude pulmonary edema: findings at high-altitude chest radiography and physical examination. Radiology. 1989; 170:661-666.

8. Bartsch P and Swenson ER. Acute high-altitude illnesses. N Engl J Med. 2013; 369:1666-1667.

9. Droma Y, Hanaoka M, Ota M, Katsuyama Y, Koizumi T, Fujimoto K, Kobayashi T and Kubo K. Positive association of the endothelial nitric oxide synthase gene polymorphisms with high-altitude pulmonary edema. Circulation. 2002; 106:826-830.

10. Qi Y, Sun J, Zhu T, Wang W, Liu J, Zhou W, Qiu C and Zhao D. Association of angiotensin-converting enzyme gene insertion/deletion polymorphism with high-altitude pulmonary oedema: a meta-analysis. J Renin Angiotensin Aldosterone Syst. 2011; 12:617-623.

11. Sartori C, Vollenweider L, Loffler BM, Delabays A, Nicod $\mathrm{P}$, Bartsch P and Scherrer U. Exaggerated endothelin release in high-altitude pulmonary edema. Circulation. 1999; 99:2665-2668.

12. Droma Y, Hayano T, Takabayashi Y, Koizumi T, Kubo $\mathrm{K}$, Kobayashi $\mathrm{T}$ and Sekiguchi M. Endothelin-1 and interleukin-8 in high altitude pulmonary oedema. Eur Respir J. 1996; 9:1947-1949.

13. Hanaoka M, Droma Y, Ota M, Ito M, Katsuyama Y and Kubo K. Polymorphisms of human vascular endothelial growth factor gene in high-altitude pulmonary oedema susceptible subjects. Respirology. 2009; 14:46-52.

14. Hanaoka M, Droma Y, Hotta J, Matsuzawa Y, Kobayashi T, Kubo K and Ota M. Polymorphisms of the tyrosine hydroxylase gene in subjects susceptible to high-altitude pulmonary edema. CHEST Journal. 2003; 123:54-58.

15. He Y, Chen J, Zhang X, Li X, Du J, He X, Zhang Z, Zhang Y, Kang L, Jin T and Yuan D. Telomere length related gene ACYP2 polymorphism is associated with the risk of HAPE in Chinese Han population. The journal of gene medicine. 2016 .
16. Stobdan T, Kumar R, Mohammad G, Thinlas T, Norboo T, Iqbal $\mathrm{M}$ and Pasha MAQ. Probable role of $\beta 2$-adrenergic receptor gene haplotype in high-altitude pulmonary oedema. Respirology. 2010; 15:651-658.

17. Buroker NE, Ning X-H, Zhou Z-N, Li K, Cen W-J, Wu X-F, Ge M, Fan L-P, Zhu W-Z, Portman MA and Chen S-H. Genetic associations with mountain sickness in Han and Tibetan residents at the Qinghai-Tibetan Plateau. Clinica Chimica Acta. 2010; 411:1466-1473.

18. He JQ, Ruan J, Connett JE, Anthonisen NR, Pare PD and Sandford AJ. Antioxidant gene polymorphisms and susceptibility to a rapid decline in lung function in smokers. Am J Respir Crit Care Med. 2002; 166:323-328.

19. Hanaoka M, Droma Y, Hotta J, Matsuzawa Y, Kobayashi T, Kubo K and Ota M. Polymorphisms of the tyrosine hydroxylase gene in subjects susceptible to high-altitude pulmonary edema. Chest. 2003; 123:54-58.

20. Liang S-Q, Chen X-L, Deng J-M, Wei X, Gong C, Chen Z-R and Wang Z-B. Beta-2 adrenergic receptor (ADRB2) gene polymorphisms and the risk of asthma: a meta-analysis of case-control studies. PloS one. 2014; 9:e104488.

21. Buchmayer H, Sunder-Plassmann G, Hirschl MM, Kletzmayr J, Woisetschlager C, Laggner AN, Horl WH and Fodinger M. G-protein beta3 subunit gene (GNB3) polymorphism $825 \mathrm{C}-->\mathrm{T}$ in patients with hypertensive crisis. Crit Care Med. 2000; 28:3203-3206.

22. Brummond NR, Saito YA, Locke GR, 3rd, Larson JJ, Atkinson EJ, Romero Y and Talley NJ. Letter: role of GNbeta3 polymorphisms in oesophageal adenocarcinoma and gastroesophageal reflux disease. Aliment Pharmacol Ther. 2015; 41:600-601.

23. Sekine A, Tanabe N, Sugiura T, Shigeta A, Jujo T, Nishimura R, Sakao S, Kasahara Y and Tatsumi K. Polymorphism of the $\mathrm{G}$ protein beta3 subunit gene influences the efficacy of sildenafil in patients with pulmonary hypertension. Internal medicine (Tokyo, Japan). 2014; 53:291-297.

24. Wang P, Koehle MS and Rupert JL. Common haplotypes in the beta-2 adrenergic receptor gene are not associated with acute mountain sickness susceptibility in Nepalese. High altitude medicine \& biology. 2007; 8:206-212.

25. Chandramoorthi GD, Piramanayagam S and Marimuthu P. An insilico approach to high altitude pulmonary edema Molecular modeling of human beta2 adrenergic receptor and its interaction with Salmeterol \& Nifedipine. Journal of molecular modeling. 2008; 14:849-856.

26. van Lieshout EM, Roelofs HM, Dekker S, Mulder CJ, Wobbes T, Jansen JB and Peters WH. Polymorphic expression of the glutathione S-transferase P1 gene and its susceptibility to Barrett's esophagus and esophageal carcinoma. Cancer Res. 1999; 59:586-589.

27. Mishra A, Ali Z, Vibhuti A, Kumar R, Alam P, Ram R, Thinlas T, Mohammad G and Pasha MA. CYBA and GSTP1 variants associate with oxidative stress under hypobaric hypoxia as observed in high-altitude pulmonary oedema. Clinical science (London, England : 1979). 2012; 122:299-309. 
28. Korzeniewski K, Nitsch-Osuch A, Guzek A and Juszczak D. High altitude pulmonary edema in mountain climbers. Respir Physiol Neurobiol. 2015; 209:33-38.

29. Wang P, Koehle MS and Rupert JL. Common Haplotypes in the $\beta$-2 Adrenergic Receptor Gene Are Not Associated with Acute Mountain Sickness Susceptibility in Nepalese. High Altitude Medicine \& Biology. 2007; 8:206-212.

30. Andreou D, Soderman E, Axelsson T, Sedvall GC, Terenius L, Agartz I and Jonsson EG. Polymorphisms in genes implicated in dopamine, serotonin and noradrenalin metabolism suggest association with cerebrospinal fluid monoamine metabolite concentrations in psychosis. Behav Brain Funct. 2014; 10:26.

31. Pereira TV, Kimura L, Suwazono Y, Nakagawa H, Daimon M, Oizumi T, Kayama T, Kato T, Li L, Chen
S, Gu D, Renner W, Marz W, Yamada Y, Bagos PG and Mingroni-Netto RC. Multivariate meta-analysis of the association of G-protein beta 3 gene (GNB3) haplotypes with cardiovascular phenotypes. Molecular biology reports. 2014; 41:3113-3125.

32. Yu D-H, Liu D-P, Wang L-Y, Chen J, Jaquish CE, Rao DC, Hixson JE, Huang J-F, Chen C-S, Gu C, Chen J-C, Cao J, Chen S-F, Whelton PK, He J and Gu D-F. Genetic variants in the ADD1 and GNB3 genes and blood pressure response to potassium supplementation. Frontiers of Medicine in China. 2010; 4:59-66.

33. Vardeny O, Peppard PE, Finn LA, Faraco JH, Mignot E and Hla KM. beta2 adrenergic receptor polymorphisms and nocturnal blood pressure dipping status in the Wisconsin Sleep Cohort Study. Journal of the American Society of Hypertension. 2011; 5:114-122. 